\title{
Niveles de contaminantes que afectan la calidad de aguas del Rio Huaura en su cuenca baja
}

\author{
Levels of pollutants that affect water quality of Huaura River in its lower basin \\ José del Carmen Cuellar Reyes ${ }^{1}$, Juan Eduardo Navarro Rojas
}

\section{RESUMEN}

Objetivo: El objetivo es realizar una caracterización de la variabilidad espacial y temporal de la calidad de las aguas del rio Huaura que permita establecer los usos del recurso más adecuado de acuerdo a las normas establecidas Métodos: Tipo de investigación descriptiva diacrónica, la caracterización se efectuó fijando tres estaciones de muestreo utilizando un Laboratorio Portátil complementando con las estaciones de monitoreo fijadas por la Dirección Regional de Salud del Gobierno Regional - DIRESA comparándolos con los límites máximos permitidos - LMP de las normas peruanas. Resultados: La Temperatura, Conductividad Eléctrica y oxígeno disuelto - OD están en rangos aceptables en acorde a la altitud y estaciones, el potencial de hidrogeno - $\mathrm{pH}$, presenta valores que tienden a rangos alcalinos, el cobre, Zinc, hierro, fierro, manganeso, plomo presenta concentraciones que sobrepasan los límites máximos establecido por la Agencia de Protección Ambiental de Estados Unidos - EPA, en cuanto al Mercurio, Plata, Molibdeno, Antimonio, Selenio, Estaño, Talio y Arsénico en todas las estaciones están por debajo de los estándares (EPA), en cuanto a los metales pesados analizados el 10/12/2013 por Dirección regional de Salud Ambiental DIRESA comparados con los estándares nacionales, el parámetro Hierro, Aluminio, Manganeso, Boro, Bario, Berilio, Calcio, Cerio, Cobalto, Potasio, Litio, Magnesio, sodio, Níquel, Fosforo, Silicio, Estroncio, Titanio, vanadio sobrepasan los (LMP) en todas las estaciones, cadmio en la estación P4, Cromo en la estación P6, molibdeno en la estación P8. Conclusiones: Comparados con los Límites máximos permisibles de la Ley General de aguas del país con la (EPA), el Arsénico, Cobre, Cromo, Fierro, Hierro total, Manganeso, Plomo, Zinc, Aluminio, Boro, Bario, Calcio, Cerio Cobalto, Mercurio, Potasio, Litio, Magnesio, Molibdeno, Sodio, Níquel, Fosforo, Silicio, Estroncio, Vanadio, Demanda Bioquímica de Oxigeno - DBO5, Demanda Química de Oxigeno - DQO, coliformes Fecales, coliformes fecales Termotolerantes están por encima de los límites máximos permitidos.

Palabras clave: Calidad de aguas, cuenca hidrográfica baja, contaminantes.

\section{ABSTRACT}

Objective: To perform a characterization of the spatial and temporal variability of the water quality of Huaura River, that allows establishing the uses of the most appropriate resource according to established standards. Methods: Type of descriptive diachronic research, the characterization was make establishing three sampling stations using a portable laboratory complementing with the monitoring stations set by the Regional Health Management of Regional Government DIRESA comparing them with the maximum limits permitted - MLP of the Peruvian norms. Results: Temperature, electrical conductivity and dissolved oxygen - $\mathrm{DO}$ are in acceptable ranges according to altitude and stations, hydrogen potential - $\mathrm{pH}$ presents values that tend to alkaline ranges, copper, zinc, iron, iron, manganese, lead present values that exceed the maximum limits established by the US Environmental Protection Agency (EPA) for mercury, silver, molybdenum, antimony, selenium, tin, thallium and arsenic in all seasons are below standards (EPA), as regards heavy metals analyzed on December 10th 2013 by Regional Environmental Health Management DIRESA, compared with the national standards, the parameter iron, aluminum, manganese, boron, barium, beryllium, calcium, cerium, cobalt, potassium, lithium, magnesium, sodium, nickel, phosphorus, silicon, strontium, titanium, vanadium exceed the MLP in all stations, cadmium at station P4, chromium at station P6, molybdenum at station P8. Conclusions: Compared with the maximum permissible limits of the General Law of the country's waters with the EPA, arsenic, copper, chromium, iron, total iron, manganese, lead, zinc, aluminum, boron, barium, calcium, cobalt cerium, mercury, potassium, lithium, magnesium, molybdenum, sodium, nickel, phosphorus, silicon, strontium, vanadium, biochemical oxygen demand - BOD5, chemical oxygen demand - COD, fecal coliforms, thermotolerant fecal coliforms are above the maximum limits allowed..

Keywords: Water quality, low watershed, pollutants.

Universidad Nacional José Faustino Sánchez Carrión. Huacho, Perú. 


\section{INTRODUCCIÓN}

La DIRESA (2000 - 2013), como parte de su programa de control y vigilancia de los recursos hídricos monitorea la cuenca del río Huaura con aproximadamente 13 estaciones desde la laguna de Patón ubicada en uno de los flancos importantes hasta el Río Huaura a la altura del Puente en el cruce con la Panamericana Norte y/o puente de Huaura con la finalidad de tener un control de la calidad de las aguas en función a la normatividad legal, Ministerio del Ambiente (2008), para lo cual han tomado muestras de parámetros físicos, químicos y biológicos teniendo como principales indicadores: $\mathrm{pH}$, temperatura, conductividad eléctrica, turbidez, oxígeno disuelto, demanda bioquímica de oxígeno, sólidos totales disueltos, Plomo, Cadmio, Cobre, Cromo, Zinc, Fierro, Arsénico. Cuellar et al. (2012) en el Proyecto de Investigación Monitoreo Comunitario para la recuperación Ambiental sostenible de la Cuenca Alta del río Huaura de la Universidad Nacional José Faustino Sánchez Carrión de Huacho evaluando las aguas del Río Patón y Quichas que conforman el río Oyón y que discurre aguas abajo hasta unirse con el río Checras; unión del Rio Churin y Chiuchin ha determinado las características principales de los ríos que conforman la cuenca Alta del rio Huaura, comparándolos con los principales protocolos y normativas de calidad de aguas para riego de vegetales y de consumo humano directo y de la EPA (2000), los resultados muestran que las aguas de estos ríos presentan serios problemas fisicoquímicos y microbiológicos de acuerdo a las normas peruanas oficiales, Ministerio del Ambiente (2008) en importantes estaciones de muestreo, lo cual les da el carácter de no aptas para actividades con contacto directo, siendo más evidentes con los estándares de la (EPA). Cárdenas,(2003), la parte baja de la Cuenca del río Huaura, desde los 2000 msnm ubicada en la zona Oeste de la Región Lima que estudiamos no está ajena a los problemas de deterioro de los cursos de agua que drena directamente al mar en la Caleta Carquín jurisdicción de la Provincia de Huaura en la costa, la que limita al Norte con las cuencas del rio Supe y Pativilca, por el Este con las cuencas de los ríos Marañón, Huallaga y Mantaro, al Oeste con el Océano Pacífico y por el Sur con la Cuenca del rio Huaral-Chancay así como las ciudades emplazadas en las riberas de ellos, con un importante aumento poblacional, es el caso de Churin, Sayán y Huaura. Estos centros urbanos vierten sus aguas servidas y hasta residuos sólidos directamente a los cursos de agua, sin tratamiento previo, lo que se traduce en incremento de aguas servidas sin tratamiento que discurren a las aguas finalmente colectándose en el rio con una enorme carga contaminante cuyas características es necesario determinar, ya que como afirma Rivera (2002), la calidad del agua es relevante por los diversos roles que esta cumple, fuera del curso del río (uso doméstico, agrícola e industrial), en el curso del río (recreación y estética) y como medio para la acuicultura y manejo de vida silvestre en general.

Se justifica la urgente necesidad de prevenir la contaminación y manejar los peligros ambientales en el río Huaura para que en el futuro no genere problemas ni peligros a la población, a la infraestructura en el entorno del rio, por lo mismo que contribuirá a su recuperación ambiental mejorando la calidad de vida de los pobladores, logrando el desarrollo sostenible (ambiental, social y económico), por su viabilidad por la disponibilidad de recursos humanos calificados en la zona de estudio que se forman en la Universidad local y en la capital, igualmente recursos materiales que se pueden adquirir con aportes de la Universidad local, Gobierno Regional, y Municipios, por el aporte tecnológico al desarrollo regional en la caracterización de la variabilidad espacial y temporal de la calidad de las aguas del rio Huaura, lograr científicamente una correcta gestión ante los peligros de contaminación para contribuir a su recuperación ambiental sostenible.

La hipótesis planteada es realizar una caracterización de la variabilidad espacial y temporal de los niveles de contaminantes para determinar la calidad de las aguas del rio Huaura que permita establecer los usos del recurso más adecuado de acuerdo a las normas establecidas.

El objetivo es realizar una caracterización de la variabilidad espacial y temporal de la calidad de las aguas del rio Huaura que permita establecer los usos del recurso más adecuado de acuerdo a las normas establecidas, dado que los monitoreos que realizan estas entidades no tienen un procesamiento científico y un análisis comparativo; con el presente trabajo de investigación se está completando estas limitaciones ya que la determinación de los niveles de contaminación de las aguas del rio 
Huaura en su Cuenca Baja se contribuye a establecer las medidas de mitigación a implementar, para lograr la recuperación ambiental para su desarrollo sostenible.

\section{MATERIAL Y MÉTODOS}

ESe ha tomado como base los puntos de monitoreo de (DIGESA), (DIRESA) que monitorea desde la laguna de Patón hasta el río Huaura a la altura del puente que cruza la Carretera Panamericana y para la presente investigación se procesa los datos de las estaciones ubicadas en la Cuenca Baja desde los 2000 (msnm).

Para la realización del estudio, se recogió los datos del monitoreo de DIGESA (2001) y monitoreo de Cuellar et al. (2012) de Las estaciones ubicadas en su Cuenca baja.

(DIGESA) y últimamente DIRESA (2009-2013), realizaron monitoreos el año 2000, 2001, 2009, 2012 y 2013 y los parámetros fueron variando, $\mathrm{pH}$, Temperatura, Conductividad, Oxígeno disuelto, sólidos totales disueltos, demanda Bioquímica de oxígeno, así como metales pesados; $\mathrm{Zn}, \mathrm{Ni}, \mathrm{Cd}, \mathrm{Cu}, \mathrm{Hg}, \mathrm{Fe}, \mathrm{Mn}, \mathrm{Pb}$ y coliformes fecales, a fines del año 2013 y procesados el año 2014 se han muestreado además Aluminio, Antimonio, Arsénico, Boro, Bario, Berilio, Calcio, Cerio, Cobalto, Cromo, Estaño, Estroncio, Fósforo, Litio, Magnesio, Molibdeno, Selenio, Silicio, Sodio, Titanio, Talio y Vanadio; en el caso de la presente investigación se tomaron los puntos de muestreo, desde Churin, Acaray, Huaura, Rio Huaura, Puente Huaura vale decir la parte de la cuencabaja.

\section{Procedimientos analíticos}

Se procesaron los parámetros monitoreados por DIGESA (2001), Rio Checras Puente Tingo, M9, Rio Checras $500 \mathrm{~m}$ aguas debajo de la Planta de filtrado de la empresa minera Izcaycruz, M10, Rio Checras 200 m aguas debajo de la Planta de filtrado de la empresa minera Izcaycruz, M11, Rio Huaura Puente Alco antes de confluencia con Rio Huaura, M12, Rio Huaura Puente Balta Sayán, M13, Rio Huaura Puente de la Carretera Panamericana, el año 2014 en las siguientes estaciones M5, Rio Checras, Puente Tingo a $100 \mathrm{~m}$ de confluencia con el río Huaura, M6, Río Huaura a $50 \mathrm{~m}$ de confluencia con el río Checras, M7, Rio Huaura, Puente Pampa Libre, M8, Río Huaura, Túnel Desembocadero Empresa Cheves, Km. 85 Carretera Huaura-Oyón, M9, Rio Huaura Puente Sayán, M10, Rio Huaura Puente Huaura.

Se procesaron los parámetros monitoreados por DIGESA (2001), analizados el 10/12/2013 comparados con el estándar de la (EPA):

Estación P4, Rio Checras, Puente de Tingo a $100 \mathrm{~m}$ de confluencia con el rio Huaura, P6, Rio Huaura a $50 \mathrm{~m}$ de confluencia con el rio Checras, P7, Rio Huaura puente Pampa libre, P8, Puente Huaura Túnel desembarcadero Empresa Cheves, Km. 85 carretera Huaura Oyón, P9, Rio Huaura puente Sayán, P10, Rio Huaura puente Huaura.

\section{RESULTADOS}

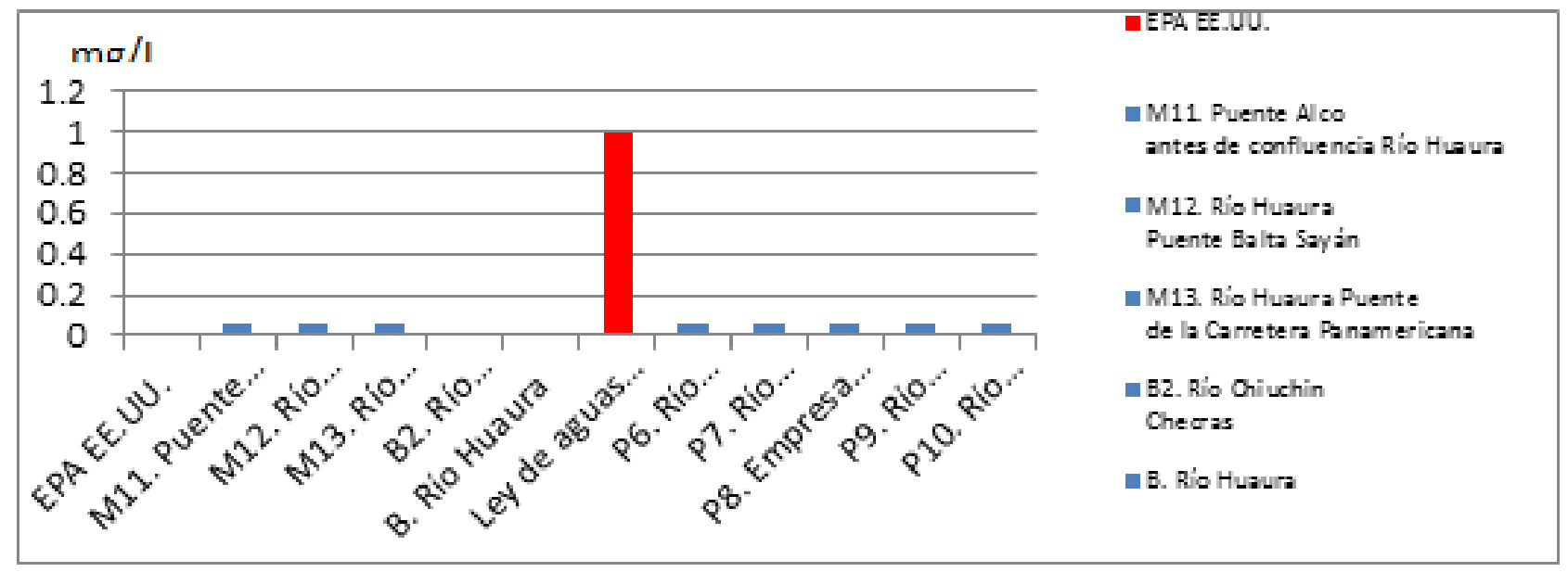

Figura 1. Resultados de concentración de Cromo

Fuente: Julio, Noviembre 2000; Setiembre, Noviembre 2001 DIGESA; 27 Noviembre 2012, Cuellar, Marzo 2013, DIRESA. 


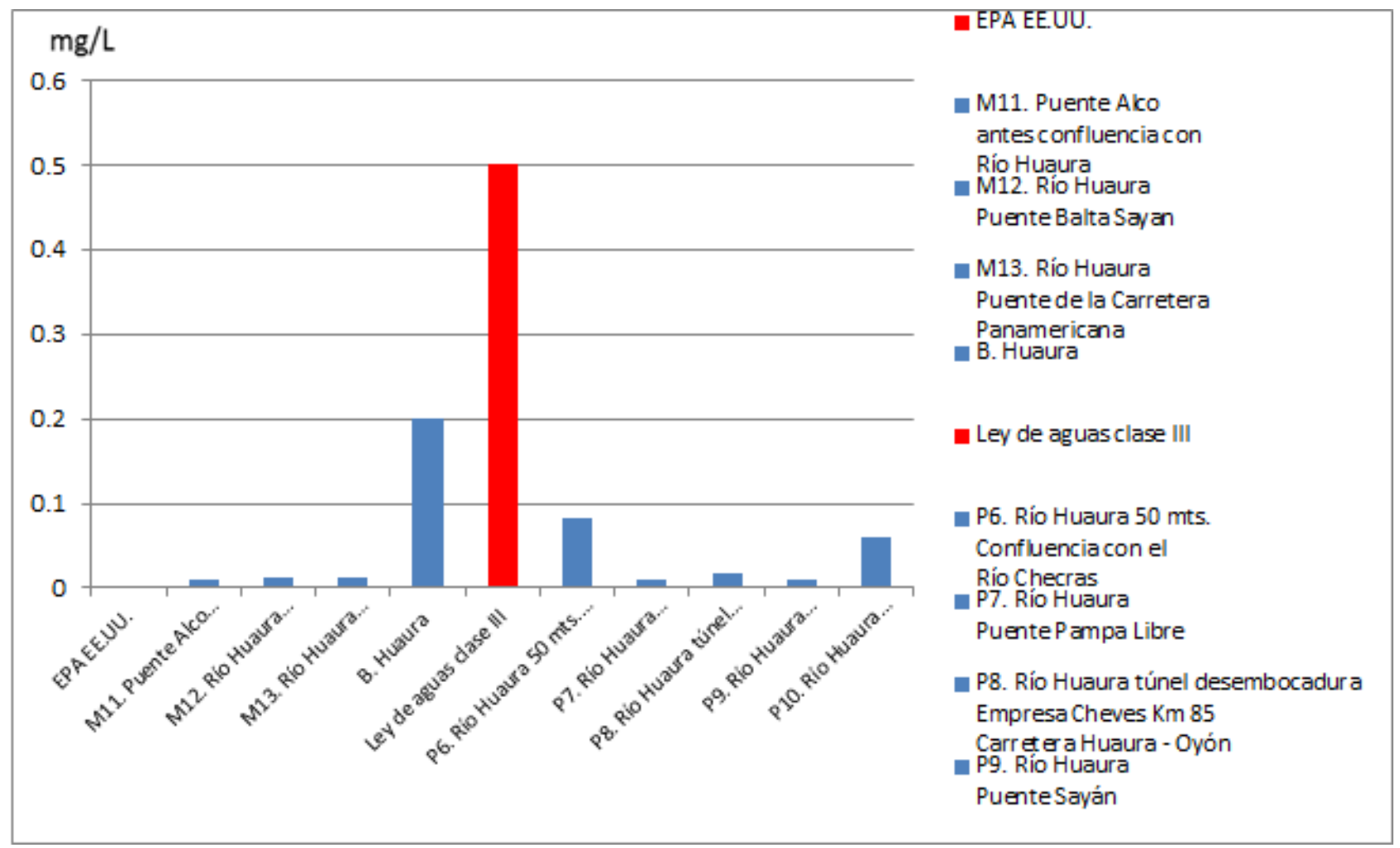

Figura 2. Resultados de concentración de cobre

Fuente: Julio, Noviembre 2000; Setiembre, Noviembre 2001 DIGESA; 27 Noviembre 2009, Cuellar, Marzo 2013, DIRESA.

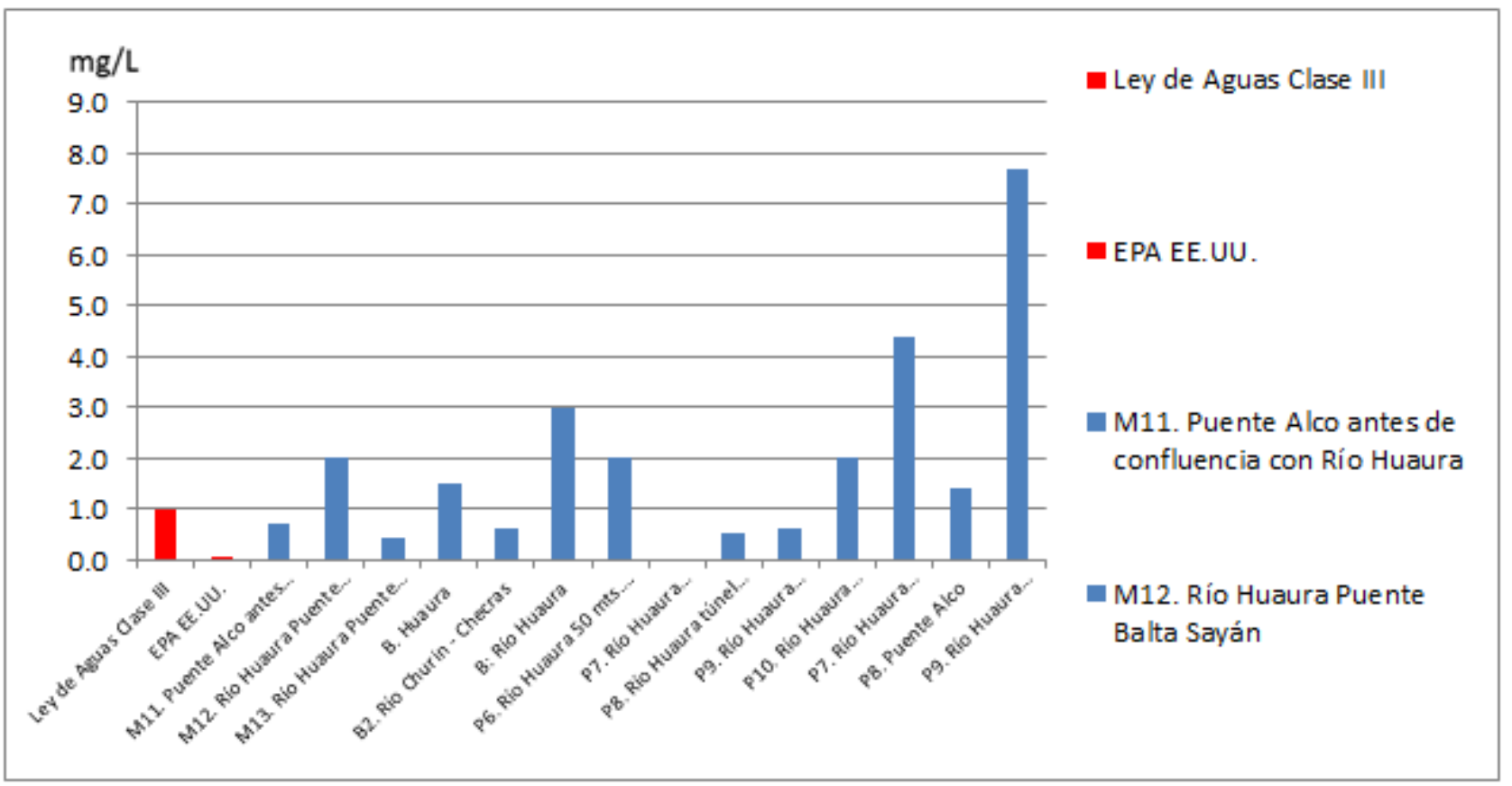

Figura 3. Resultados de concentración de Fierro

Fuente: Julio, Noviembre 2000; Setiembre, Noviembre 2001 DIGESA; 27 Noviembre 2012, Cuellar, Marzo, Diciembre 2013, DIRESA. 


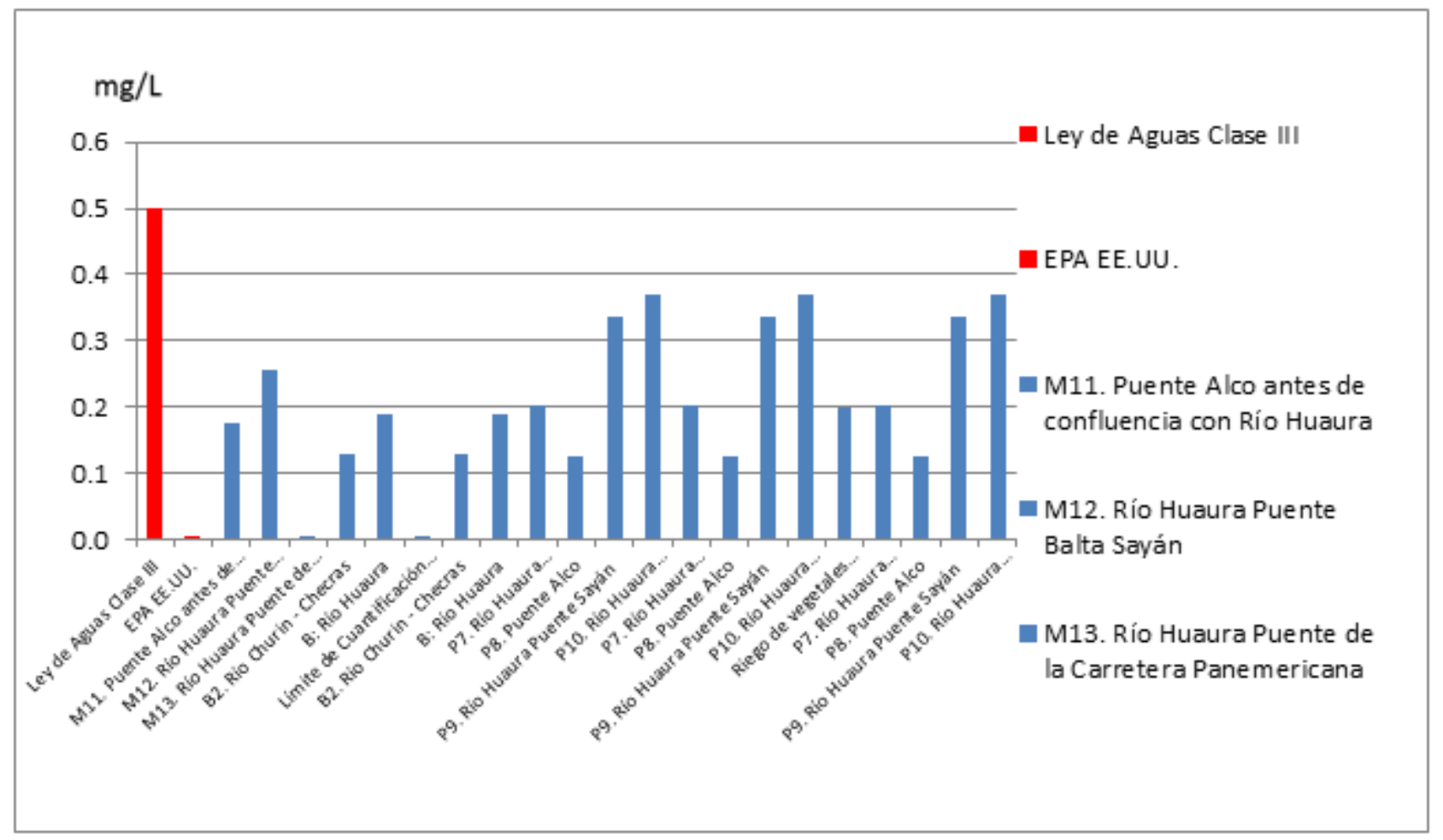

Figura 4. Resultados de concentración de Manganeso

Fuente: Julio, Noviembre 2000; Setiembre, Noviembre 2001 DIGESA; 27 Noviembre 2012, Cuellar, Marzo, Diciembre 2013, DIRESA.

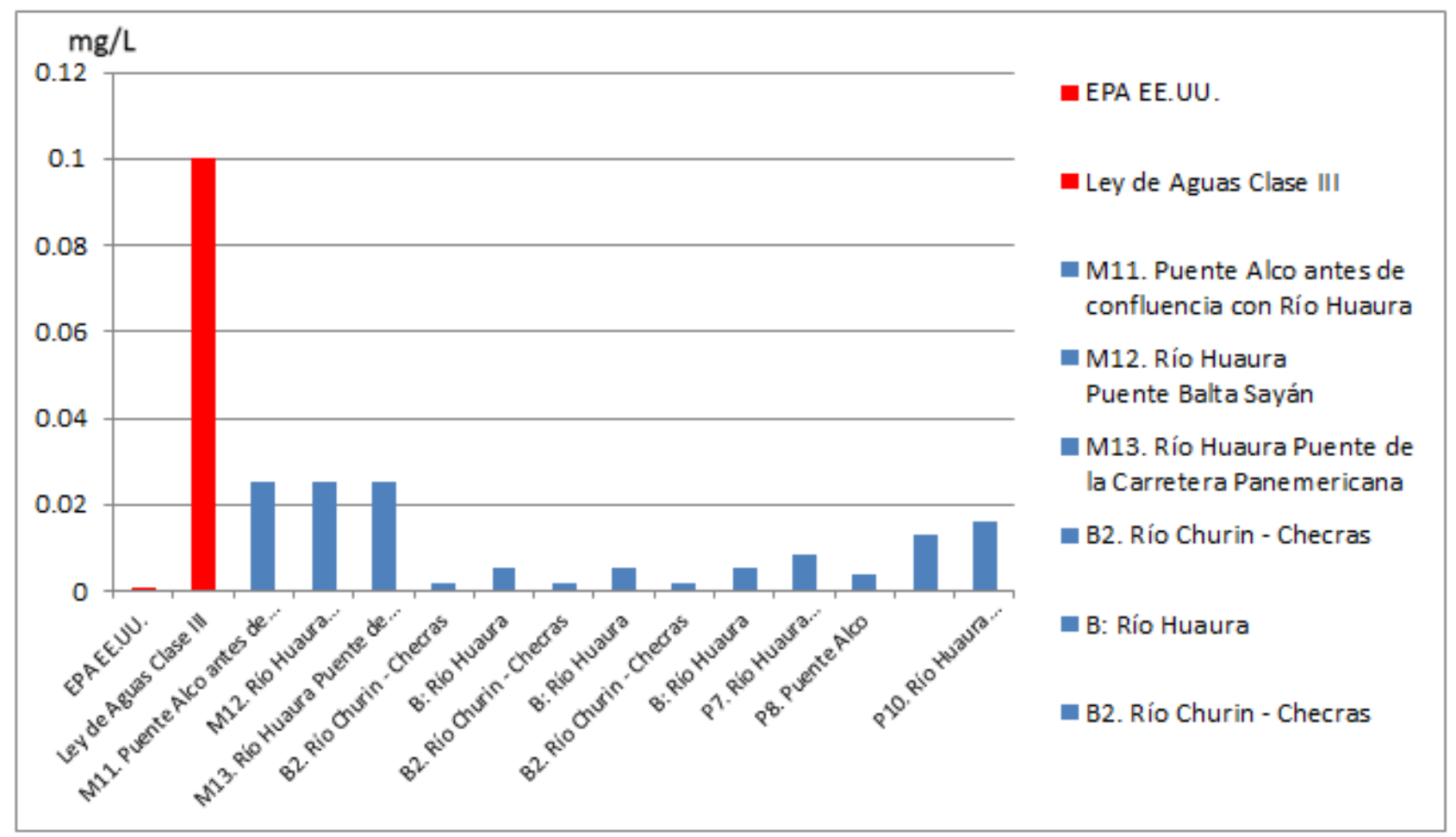

Figura 5. Resultados de concentración de Plomo

Fuente: Julio, Noviembre 2000; Setiembre, Noviembre 2001 DIGESA; 27 Noviembre 2012, Cuellar, Marzo, Diciembre 2013, DIRESA. 


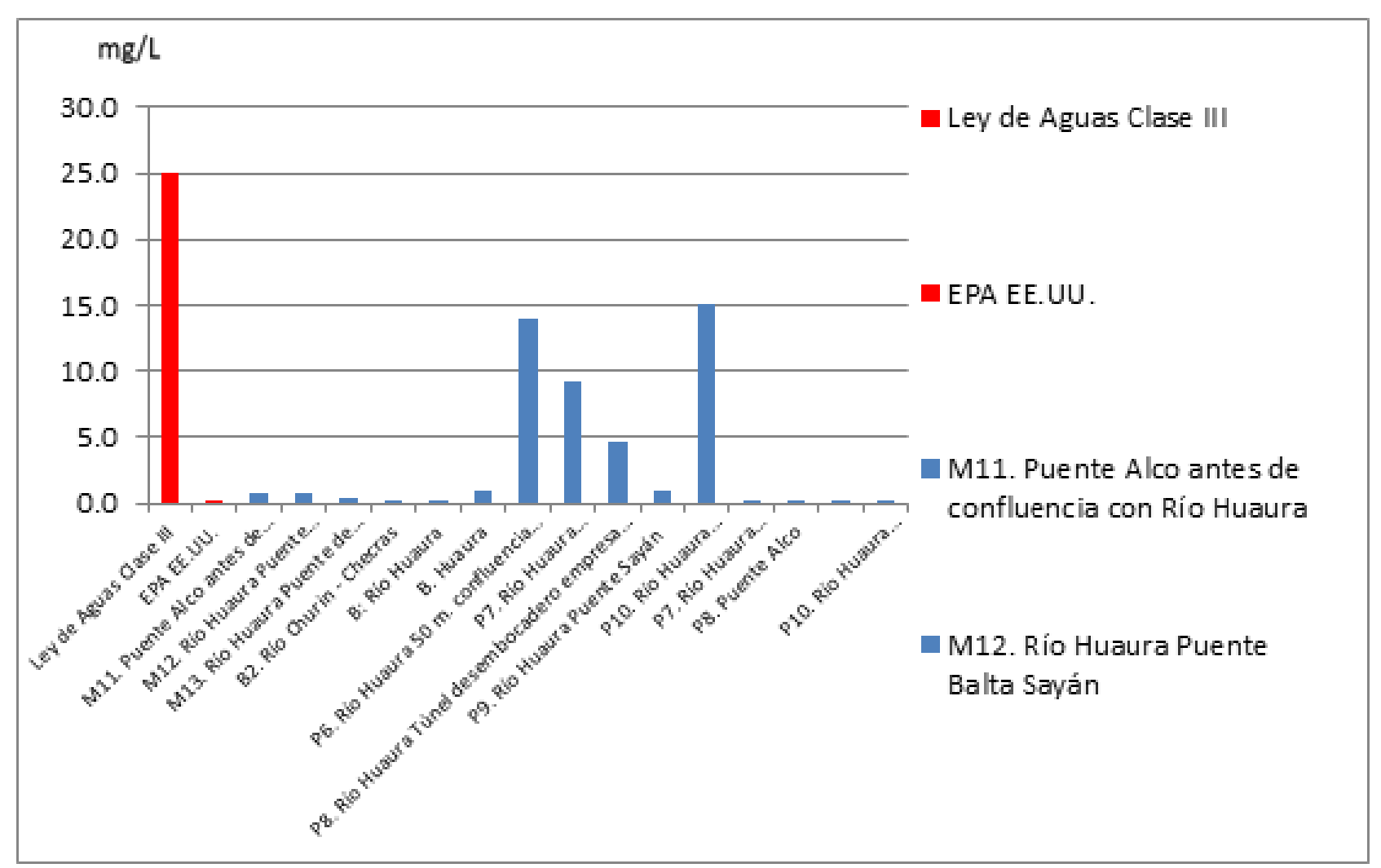

Figura 6. Resultados de concentración de Zinc

Fuente: Julio, Noviembre 2000; Setiembre, Noviembre 2001 DIGESA; 27 Noviembre 2012, Cuellar, Marzo, Diciembre 2013, DIRESA

En cuanto al Cromo (Figura 1), Cuellar et al. (2012) en las tres estaciones muestreadas en la Estación B2 río Chiuchin y B en Tingo cabecera de la Cuenca Baja sobrepasan el límite máximo establecido por la (EPA). (DIGESA) y DIRESA (2009-2013), registra valores por debajo del (LMP) de la clase III de la Ley de Aguas, el Cromo solo en la estación $\mathrm{P} 6$ registrado el 10/12/2013 no supera el (LMP) según el estándar de la (EPA).

En cuanto al Cobre, Figura 2, los ríos estudiados presentaron concentraciones de cobre entre 0,04 y $0,58 \mathrm{mg} / \mathrm{L}$ el límite superior sobrepasa los LMP, (DIGESA) en todas sus estaciones de monitoreo no registra valores por encima de los (LMPs), al igual que los registros de DIRESA (2009-2013) los años 2009, 2012 y 2013 no son significativos, pero el registrado el 10/12/2013 comparado con el estándar de la (EPA) de 0,0004 mg/L en las estaciones P4,P5,P6,P7,P8,P9,P10 los valores registrados sobrepasan largamente este estándar y consecuentemente en lo anteriores registros reseñados.

En cuanto al Zinc, Figura 6, con el laboratorio Portátil Cuellar et al. (2012) los valores encontrados fueron entre 0,07 y $1,14 \mathrm{ppm}$ y
0,07 y $1,52 \mathrm{mg} / \mathrm{L}$ resulta aceptable por cuanto la norma peruana para agua potable acepta hasta 3 ppm, los resultados por los laboratorios certificados todas sobrepasan los límites máximo establecido por la (EPA). Los resultados de (DIGESA) no sobrepasan los (LMPs) en las estaciones que han monitoreado. En los de DIRESA (2009-2013) los años 2009, 2012 y 2013, la mayoría de estaciones sobrepasan los (LMPs), Y en cuanto a los registros de (DIRESA) del 10/12/2013 comparados con el estándar (EPA) de 0.003 $\mathrm{m} / \mathrm{L}$, las estaciones ubicadas en la cuenca baja P4, P5, P6, P7, P8, P9, P10 sobrepasan largamente este estándar y consecuentemente a los registros anteriores citados.

En cuanto al Fierro, Figura 3, las aguas estudiadas presentaron concentraciones de fierro que oscilaron entre los 0,11 y $3,3 \mathrm{mg} / \mathrm{L}$ Las concentraciones son superiores al estándar de calidad de aguas de regadío ( $1 \mathrm{mg} / \mathrm{L})$, muy superiores a aguas para uso potable $(0,3 \mathrm{mg} / \mathrm{L})$ y $(2 \mathrm{mg} / \mathrm{L})$ para efluentes mineros según Norma Peruana. (DIGESA) solo monitorea el mes de setiembre del año 2001, en el mismo ámbito en las estaciones M4, M6, M7, M8 y M12 sobrepasan los (LMP). En cuanto a los registros de DIRESA (2009-2013) los años 2009 y 2012 
no se monitorea este parámetro, y en el muestreo del año 2013, las estaciones P6 y P10 sobrepasan los límites permitidos, en cuanto al Hierro, los resultados del Laboratorio certificado todas las estaciones sobrepasan los límites máximos establecidos por la (EPA) y en cuanto a los registros de (DIRESA) el año 2013 al igual que los registros del 10/12/2013 en todas las estaciones ubicadas en la cuenca baja, están por encima de los (LMPs).

En cuanto al Manganeso, Figura 4 los resultados por el laboratorio certificado todas sobrepasan los límites máximo establecido por la (EPA), (DIGESA) solo monitorea el mes de setiembre del 2001, registrando valores por encima de los LMP en las estaciones M4, M5, M6 y M7 (DIRESA) en Marzo del año 2013 registra valores altos en las estaciones P9 y P10 y en Diciembre del 2013 al igual que los registros con el estándar de la (EPA) todas las estaciones registran valores por encima de los (LMPs).

En cuanto al Plomo, Figura 5 los resultados por Cuellar et al. (2012) en tres estaciones, se observa que la Estación B río Huaura presenta valores por encima del límite establecido, así como en las tres estaciones B, B1, B2 presente tenores por encima del límite establecido, (DIGESA) en sus 4 monitoreos de los años 2000 y 2001 registra valores por encima de los (LMP) solo el mes de julio del 2000 en las estaciones M4, M5, M6, M7 y M8, (DIRESA) registra en todos los años que ha muestreado valores por debajo de los estándares permitidos, en cuantos a los resultados del 10/12/2013 comparados con los (LMP) de la (EPA) de $0,0004 \mathrm{mg} / \mathrm{L}$ todas las estaciones ubicadas en la Cuenca Baja sobrepasan estos límites.

En cuanto al Mercurio, los resultados por el laboratorio certificado todas no determinan presencia de este metal, al igual que en los de (DIGESA) y DIRESA (2009-2013), en cuantos a los resultados del 10/12/2013 comparados con los LMP de la EPA de $0,001 \mathrm{mg} / \mathrm{L}$ solo la estación P4 sobrepasa este límite.

En cuanto a los 32 metales pesados analizados el 10/12/2013 comparados con los estándares nacionales, el $\mathrm{Ph}$ de las estaciones ubicadas en la Cuenca Baja puntos P4, P5, P6, P7, P8, P9 y P10 para el parámetro Hierro según (DIRESA) muestran valores que exceden el (ECA)-Agua
Categoría 3, al igual que en el punto P9 para el parámetro Aluminio así mismo en los puntos $\mathrm{P} 5, \mathrm{P} 6, \mathrm{P} 9, \mathrm{P} 10$ para el parámetro Manganeso.

\section{DISCUSIÓN}

Los resultados promedios de los parámetros analizados presentados en graficas según estaciones muestreadas y mes realizado, comparados con los (LMP), analizados por los laboratorios certificados, Cuellar et al. (2012), tales como el Cobre, presentaron valores por debajo de los (LMPs) al igual que de los de (DIGESA), pero el registrado el 10/12/2013 comparado con el estándar de la (EPA) de 0,0004 mg/L en las estaciones P4, P5, P6, P7, $\mathrm{P} 8, \mathrm{P} 9, \mathrm{P} 10$ superan largamente este estándar y consecuentemente a los anteriores registros señalados. El zinc no se considera tóxico, aunque confiere al agua un sabor desagradable. En aguas saladas y dulces es muy estable (Rivera y Muñoz-Pedreros (1999), los valores encontrados por Cuellar et al. (2012) fueron entre 0,031 y 0,93 ppm. Valores por encima de los estándares de la (EPA), en cambio (DIGESA) y (DIRESA) no registra valores por encima de los (LMP), pero el registrado el 10/12/2013 comparado con el estándar de la (EPA) de $0,0004 \mathrm{mg} / \mathrm{L}$ en las estaciones P4, P5, P6, P7, P8, P9, P10 superan largamente este estándar; estas en altas concentraciones reducen el crecimiento de la planta y provoca acumulaciones indeseables en los tejidos. De acuerdo a las investigaciones realizadas se reconoce que el Zinc se acumula irreversiblemente en el suelo. Por ello, las aplicaciones en exceso de lo requerido por las plantas, eventualmente llegan a contaminar los suelos, los cuales pueden convertirse en suelos no productivos o producir cosechas inaceptables. Los valores encontrados por Cuellar et al. (2012) fueron entre 0,07 y 1,01 $\mathrm{mg} / \mathrm{L}$ resulta aceptable por cuanto la norma peruana para agua potable acepta hasta $3 \mathrm{ppm}$. Los valores hallados por (DIGESA) no sobrepasan los (LMPs.) el Zinc forma parte de más de 100 metal enzimas (enzimas con un mineral en su estructura). Es esencial en la síntesis del Ácido desoxirribonucleico DNA, en procesos implicados en la inmunidad, curación de las heridas, percepción del sentido del gusto, síntesis de esperma y el desarrollo físico normal, en cuanto a los resultados de los laboratorios certificados Cuellar et al. (2012), todos sobrepasan el límite máximo establecido por la (EPA). 
En cuanto al Fierro Cuellar et al. (2012), presentaron concentraciones de fierro que oscilaron entre los 0,11 y 3,3 mg/L (DIGESA) solo monitoreo el mes de setiembre del 2001 hallando valores por encima de los (LMPs) en las estaciones M4, M6, M7, M8 y M12, las concentraciones son superiores al estándar de calidad de aguas de regadío ( $1 \mathrm{mg} / \mathrm{L}$ ), muy superiores a aguas para uso potable $(0,3 \mathrm{mg} / \mathrm{L})$ y $(2 \mathrm{mg} / \mathrm{L})$ para efluentes mineros según Norma Peruana, el río que presentan el nivel más alto es, Huaura sobrepasando los (LMP) para uso potable, de riego de vegetales y de efluentes mineros, en cuanto a los valores hallados por DIRESA (2009-2013) en el año 2013, dos estaciones sobrepasan los (LMPs.) El hierro puede depositarse como hidróxido y obturar las branquias de los organismos, disminuyendo su potencial respiratorio. Los huevos y las larvas cultivadas son más sensibles a estos metales, produciéndose entonces su muerte, Rivera y Muñoz-Pedreros (2004), el fierro en el medio acuático no es nocivo al estar en bajo contenido, pero suele serlo en presencia de altas concentraciones, para las truchas, con valores de $\mathrm{pH}$ en agua de 6,5 a 7,5 y concentraciones de $0,9 \mathrm{ppm}$ de hierro, es de efecto mortal, Rivera y Muñoz-Pedreros, (2002), el ingreso a los cultivos se produce a través del agua de abastecimiento y como consecuencia de operaciones industriales efectuadas por el hombre (papeleras, extracción de minerales, curtiembres, etc.) Bien porque son producto del arrastre de las aguas. El principal problema radica en que son bioacumulables, afectan el sistema neuromuscular y pueden llegar a ser letales, Rivera y Muñoz-Pedreros (2004).

En cuanto al Hierro, (DIGESA) no monitorea este elemento, los valores registrados para Cuellar et al. (2012), por los laboratorios certificados todas sobrepasan los límites máximos establecidos por la (EPA), (DIRESA) registra en el mes de Diciembre del año 2013 valores de 7,71 a 7,701 valores sumamente altos, considerando que es un elemento esencial para los cultivos, las plantas no pueden realizar su ciclo vital en su ausencia, ya que está involucrado en el metabolismo de la planta de una manera específica. Está involucrado en la síntesis de clorofilas, y participa en un buen número de sistemas enzimáticos importantes para el metabolismo de las plantas, su deficiencia se denomina clorosis férrica y se caracteriza, de forma visual, por un amarilleamiento intervenal de las hojas jóvenes, como consecuencia de la clorosis férrica, las plantas se desarrollan mal, teniendo menor vigor y una menor producción, la clorosis es consecuencia del efecto que distintos factores, tienen sobre la absorción y distribución de hierro por las plantas y que es debido a la suma de varios procesos, estos procesos, se dan a una velocidad suficiente como para suplir las necesidades férricas de la planta y son: Solubilización de los oxihidróxidos de hierro de los suelos, por lo general muy insolubles, tanto más cuanto más elevado es el $\mathrm{pH}$ del suelo.

Transporte de Fe soluble hacia las raíces: Este transporte viene ralentizado por las bajas concentraciones de $\mathrm{Fe}$ y por las retenciones que este elemento sufre sobre distintos materiales edáficos. La presencia de transportadores sería muy beneficiosa, la absorción de Hierro por las raíces jóvenes de las plantas, este proceso está muy influenciado en por el $\mathrm{pH}$, el Bicarbonato y presencia de caliza del suelo, de manera general las plantas son capaces de reducir el Fe (III) en la superficie de la raíz y formar Fe (II) que es la especie química que las plantas pueden tomar, en cantidades excesivas reducen el crecimiento y provocan acumulaciones indeseables en los tejidos; la elevada cantidad de Hierro en el agua influye en la apariencia, el sabor, el olor o perjudicar otros usos del agua (según guías de la Organización Panamericana de Salud OPS y Organización Mundial de Salud OMS), el daño que estarían efectuando es bastante peligroso acorde a los resultados registrados.

En cuanto al Arsénico total, un importante contaminador de cultivos, en algunos casos, niveles de Arsénico tan bajos como 0,7 ppm pueden reducir el rendimiento de los cultivos en un $50 \%$ (Peterson 1980), aún en concentraciones pequeñas puede acumularse en el organismo y producir intoxicación crónica, sus síntomas son animales deprimidos, sin apetito, débiles y torpes, con temblores, convulsiones, diarreas y gastroenteritis hemorrágica, produce problemas de constipación crónica, aunque pequeñas concentraciones le confieren al agua sabor desagradable lo que limita el consumo por parte de los animales, los más susceptibles son los más jóvenes, la máxima concentración soportable por el vacuno, según distintos autores se estima de 0,15 a $0,30 \mathrm{mg} / \mathrm{L}$ pero aún con estas concentraciones se pueden producir intoxicaciones crónicas, lo cual es sumamente peligroso ya que los resultados de la presencia de Arsénico total según los Laboratorios 
Certificados en las tres estaciones registradas en todas se sobrepasa el límite máximo establecido por el laboratorio Rovill. DIGESA no monitorea este elemento. Los valores hallados por DIRESA están en los rangos permitidos hasta antes del 10/12/2013 no así en las Estaciones P7, P8, P9, P10.

Respecto al Cromo, está en cantidades excesivas reduce el crecimiento y provocan acumulaciones indeseables en los tejidos, según estudios realizados por la FAO el cromo se fija y se acumula irreversiblemente en el suelo, por ello el exceso de lo requerido por las plantas eventualmente llegan a contaminar los suelos. El Cromo (VI) es no esencial y tóxico. Los compuestos son corrosivos, y la piel alérgica puede tener reacciones justo después de que la exposición tenga lugar, independientemente de la dosis, en el agua los niveles naturales de cromo son bajos alcanzando en los ríos concentraciones que varían entre 0,1 a $5 \mathrm{mg} / \mathrm{L}$ la Agencia Internacional para la Investigación de Cáncer (IARC) clasifica a los compuestos de cromo (VI) como cancerígenos conocidos. La exposición profesional a largo plazo al cromo transportado por el aire a niveles más altos que los que hay en el ambiente natural se ha asociado con el cáncer de pulmón. (Cousillas, 2005). Resultados de la presencia de Cromo total según los Laboratorios Certificados son limitados ya que en las tres (3) estaciones muestreadas solo en la Estación B2 río Chiuchin y $B$ en Tingo sobrepasan el límite máximo establecido por la (EPA). (DIGESA) y (DIRESA) registran valores por debajo del (LMP) de la clase III de la Ley de Aguas, el Cromo solo en la estación P6 registrado el 10 de diciembre del 2013 no supera el (LMP) según el estándar de la (EPA).

En cuanto al Manganeso, este puede causar síntomas de toxicidad y deficiencia en plantas, cuando el $\mathrm{pH}$ del suelo es bajo las deficiencias de Manganeso son más comunes, tal que concentraciones altamente tóxicas de Manganeso causan inflamaciones de las paredes celulares, abrasamiento de las hojas y puntos marrones en las hojas, en cantidades excesivas reducen el crecimiento y provocan acumulaciones indeseables en los tejidos, como resultado de las investigaciones realizadas por la Organización de las Naciones Unidas para la Alimentación y la Agricultura FAO, reconoce que la mayoría de los oligoelementos se fijan y se acumulan, irreversiblemente en el suelo, el exceso de lo requerido por la planta eventualmente llegan a contaminar a los suelos, los cuales pueden convertirse en suelos improductivos o producir cosechas inaceptables y esto es sumamente grave ya que los resultados por los laboratorios certificados todas sobrepasan el límite máximo establecido por la (EPA), DIGESA (2001) en un solo monitoreo observado en setiembre del 2001 registra resultados por encima de los LMP en las estaciones M4, M5, M6 y M7, DIRESA (2009-2013) en todas sus estaciones de Diciembre del año 2013 registra valores por encima de los estándares.

En cuanto al Plomo, Cousillas (2005), el envenenamiento por plomo es una intoxicación del sistema por medio de compuestos orgánicos que contienen plomo, estos penetran en el cuerpo por respiración (polvo, humo o spray) o por ingestión de comida u otras sustancias que contengan plomo. Las áreas urbanas y cercanas a carreteras han sido la principal fuente de $\mathrm{Pb}$ Antropogénico, como resultado, la aportación primaria de plomo al medio ambiente se hizo a través de la combustión de gasolinas de automóviles. Según Clark, (1997), las actividades humanas liberan mucho más $\mathrm{Pb}$ a la atmósfera $(449,000$ toneladas/año) que las fuentes naturales (19,000 toneladas/año). Prueba de ello, es que las concentraciones de $\mathrm{Pb}$ están mucho más elevadas en el suelo y organismos cercanos a carreteras donde la densidad de tráfico es alta.). La contaminación del agua por plomo no se origina directamente por el plomo sino por sus sales solubles en agua que son generadas entre otras por actividades mineras, las dos principales vías de acceso de los compuestos de Plomo al organismo son el tracto gastrointestinal y los pulmones, cerca del $10 \%$ del Plomo ingerido es excretado en la orina y en menor cantidad en el sudor, en el pelo y en las uñas, el $90 \%$ del Plomo que se encuentra en el cuerpo humano se deposita en el esqueleto óseo y es relativamente inerte, y el que pasa a través del torrente sanguíneo puede depositarse en los tejidos, el Plomo que penetra en los sistemas fluviales se retira pronto del agua superficial hacia los sedimentos, absorbiéndose a la materia orgánica y arcillas, precipitándose más tarde como sales insolubles (Carbonato, Sulfato Y Sulfuro de $\mathrm{Pb}$ ), en los sedimentos reacciona con iones $\mathrm{OH}_{-}$, aluminio y óxido de magnesio. Se acumula básicamente en las capas más superficiales.

El envenenamiento debido al plomo constituye una posibilidad si la concentración de este en la 
sangre es de 0,8 partes por millón en los adultos o 0,4 partes por millón en los niños, Robertson, (1988), puesto que el plomo es un veneno acumulativo, es importante investigar continuamente las cantidades de este metal que entran al medio ambiente, que constituye una amenaza para la salud humana, (Dickson, 1980).

En cuanto a los resultados de los laboratorios certificados Cuellar et al. (2012), se realizó en tres estaciones, en el mismo se observa que la Estación B río Huaura presenta valores por encima del límite establecido, en cuanto al laboratorio Envirolab tres (3) estaciones B, B1, B2 presenta tenores por encima del límite establecido por la (EPA), (DIGESA), DIGESA (2001) registra en las Estaciones M4, M5, M6, M7, M8 solo en Julio del 2000 en las demás están por debajo del límite establecido por la Ley de aguas Clase III. (DIRESA) registra en todas sus estaciones valores por debajo de los estándares hasta antes del 10 de diciembre del 2013 en que todas las estaciones registran valores por encima del límite establecido por la (EPA).

Finalmente es importante señalar que los registros de fines del año 2013 por (DIRESA) de otros metales pesados no muestreados en anteriores monitoreos superan los (LMP) de la (EPA), de los 32 metales pesados analizados el 10 de diciembre del 2013 el Boro, Bario, Berilio, Calcio, Cerio, Cobalto, Potasio, Litio, Magnesio, sodio, Níquel, Silicio, Estroncio, Titanio, vanadio sobrepasan en todas las estaciones estos límites, , Cadmio en la estación P4, Cromo en la estación P6, Molibdeno en la estación P8 y Plata, Antimonio, Selenio, Estaño, Talio no sobrepasan en ninguna estación, Arsénico en las estaciones P4,P5,P6 de la Cuenca Baja.

\section{AGRADECIMIENTOS}

Los autores agradecen el aliento de las organizaciones representativas sociales a seguir investigando en la Cuenca del Huaura pese al poco apoyo con que cuentan los investigadores en la Región.

\section{REFERENCIAS BIBLIOGRÁFICAS}

Cárdenas, J. (2003). La calidad de las aguas para estudiantes de Ciencias Ambientales. Universidad Detrital, Colombia.

Clark, R. B., Frid, C. and Attrill, M. Marine Pollution, 4th edit. Oxford University Press, Oxford, pp. 61-79, 1997.
Cousillas C, A (2005). Contaminación de las aguas. Hytsa.

Cuellar, Chinga, Llanos (2012) Inédito Monitoreo Comunitario para la Recuperación Sostenible de la Cuenca Alta del río Huaura de Huacho. Vicerrectorado de Investigación, UNJFSC.

Dickson, T. R. (1980). Química: enfoque ecológico. Limusa.

Ministerio de Salud (2001) Dirección General de Salud Ambiental - DIGESA Dirección Regional de Salud Ambiental- DIRESA (2009-2013) Registros de Monitoreo.

EPA. (2000). Concentrations of Lead in Blood. U. S. Environmental Protection Agency. http://yosemite.epa.gov/OCHP/OCHP

Ministerio del Ambiente (2008, Julio, 31). D.S. $\mathrm{N}^{\circ}$ 002-2008-MINAM. Aprueba los Estándares nacionales de calidad ambiental para agua. Diario Oficial El Peruano, 377222 - 377227.

Peterson, B. J. (1980). Aquatic primary productivity and the $14 \mathrm{C}-\mathrm{CO} 2$ method: a history of the productivity problem. Annual Review of Ecology and Systematics, 11(1),

Rivera, N. R., Muñoz-Pedreros, A., \& Encina, F. (2002). La calidad físico química de las aguas en la reserva nacional Malleco en la IX Región de Chile. Información Tecnológica, 13(6), 37-45.

Rivera y otros (2004) La Calidad de las Aguas en los Ríos Cautín e Imperial, IX Región Chile Water Quality in the Cautín and Imperial Rivers, IX Región-Chile. Univ. Católica de Temuco, Fac. de Ciencias, (1) Dpto. de Ciencias Biológicas y Química, (2) Esc. de Ciencias Ambientales, Casilla 15-D, Temuco-Chile.

Robertson, W. O., \& Dreisbach, R. H. (1988). Toxicología clínica. Editorial el Manual Moderno, México, D.F.: México, 205-231.

\section{Correo electrónico:}

josecuellarreyes@yahoo.com.pe.

Revisión de pares:

Recibido: 18-10-2016

Aceptado: 23-12-2016 